\title{
Efficacy of Antioxidants in Amelioration the Toxic Effect of Oxidative Stress Induced By COVID- 19 Infection
}

\author{
Said Said Elshama* \\ Department of Forensic Medicine and Clinical Toxicology, College of Medicine, Suez Canal \\ University, Ismailia City, Egypt. \\ College of Medicine, Taif University, Taif, Saudi Arabia.
}

*Corresponding Author: Said Said Elshama, Department of Forensic Medicine and Clinical Toxicology, College of Medicine, Suez Canal University, Ismailia City, Egypt. College of Medicine, Taif University, Taif, Saudi Arabia.

\begin{abstract}
Pathological features of COVID-19 infection emerge usually from an imbalance between the reactive oxygen species and antioxidant system leading to toxic oxidative stress. Moreover, the oxidative stress index identifies the association between the severity of COVID-19 disease and oxidative stress level predicting the risk factors that determine the degree of severity as well as other factors that may reduce this severity. Thus, this index may be used to predict the suitable and effective treatment of COVID-19 cases. Until now, there is no specific therapy for COVID-19 cases but there are many attempts to use endogenous and natural antioxidants as complementary and alternative medicine in the treatment of COVID-19 cases such as glutathione, vitamins $C$ and $E$, antioxidants enzymes (superoxide dismutase and catalase), herbal medicine, nutraceuticals besides some trace elements such as zinc and selenium preventing lipid peroxidation and tissues damage. So, it should conduct further clinical trials on the human to assess the efficacy of antioxidant therapeutic options and its harmful effects if it is present.
\end{abstract}

Keywords: COVID-19 - Oxidative stress- Antioxidant - Treatment

\section{INTRODUCTION}

Coronavirus is considered the largest known RNA virus genome wherein it is a singlestranded RNA with 32 kilobases long. Moreover, coronavirus can multiply and change at a very high rate because it has the highest frequency to recombine and replicate from any single positive RNA strand virus leading to serious challenges during its diagnosis and treatment. This novel coronavirus was detected in China at the end of 2019 when it caused a large number of fatal pneumonia cases leading to mass disaster wherein World Health Organization (WHO) classified this outbreak as COVID-19 pandemic [1].

It is settled that most of the RNA viruses such as coronaviruses may be trigger factors for the oxidative stress process that may be responsible for most of the pathogenesis that is caused by these viruses. Noteworthy, there are oxidant agents and antioxidant system in the body wherein free radicals, reactive oxygen and nitrogen species are considered oxidant agents that are neutralized by antioxidant enzymes and agents preventing its harmful effects on the body [2]. Therefore, the imbalance between oxidant agents and the antioxidant system leads to increased production of free radicals in concomitant with antioxidants depletion causing toxic oxidative stress that is involved in RNA viruses' infection such as coronaviruses. Moreover, penetration of RNA viruses into cells causes mitochondrial dysfunction that is a source for the release of reactive oxygen species besides cytokine storms that stimulates nitric oxide production that is considered one of the reactive nitrogen species [3].

Worthwhile, reactive oxygen and nitrogen species are considered useful natural byproducts under physiological conditions regulating growth factors and cytokines, but it is considered toxic free radicals causing oxidizing different molecules such as DNA, phospholipids, and proteins during oxidative stress process. So, toxic free radicals cause lipid peroxidation and DNA damage leading to abnormal immune response and apoptosis that are involved in the pathogenesis of many diseases [4]. However, the toxic effect of these free radicals may be neutralized by antioxidants such as glutathione, vitamins such as $\mathrm{C}$ and $\mathrm{E}$, enzymes such as glutathione peroxidase, 
superoxide dismutase, and catalase besides some trace elements such as zinc and selenium preventing lipids peroxidation and then tissues damage [5].

In this context, the non-invasive oxidative stress index used to identify the association between the severity of COVID-19 disease and oxidative stress level wherein it can predict the risk factors that determine the severity degree of Covid-19 as well as other factors that may reduce this severity. Thus, this index may be used to predict the suitable and effective treatment of COVID-19 cases [6].

Therefore, the above-mentioned pathological process may have an important role in inducing the pathological features of Covid-19 as one of the RNA viruses that cause oxidative stress and then if the mode of action of the designed therapeutic protocols for the Covid-19 cases is based on this process, it may be effective and curative. Until now, there is no specific antiviral therapy for the treatment of COVID-19 cases but there are many attempts to use some therapeutic protocols and create specific vaccines. However, there are other attempts to use complementary and alternative medicine such as herbal medicine as a substitute or supportive option in the treatment of COVID-19 cases to counteract its symptoms or to decrease the viral load. So, there is an urgent necessity to evaluate the efficacy of these therapeutic options and their harmful effects if it is present.

\section{PATHOGENESIS OF COVID-19 INFECTION}

In more detail but in a brief narrative way at the same time, we would show the base of COVID19 infection pathogenesis to identify the role of toxic oxidative stress-inducing pathological features of this pandemic. Initially, it is known that the main life-threatening pathological feature of COVID-19 infection is lung inflammation that results from the liberation of a lethal storm of cytokine. This harmful inflammatory process is initiated via COVID-19 penetration into the cell inactivating Angiotensin Converting Enzyme 2 (ACE2) receptor that is localized with the Angiotensin-Converting Enzyme (ACE) at the cell surface wherein they constitute the renin-angiotensin system [7].

Moreover, ACE2 can hold the activity of ACE via decreasing the angiotensin-II and increasing the ang (1-7) peptide wherein they have antagonistic effects on each other. In addition, activation of ACE leads to oxidative stress, inflammation, and apoptosis while ACE2 activation causes anti-oxidative, antiinflammatory, and anti-apoptotic effects. Thus, the activity of ACE means an increase in the production of reactive oxygen species leading to cell damage while the activity of ACE2 leads to pro-oxidant suppression preventing the cellular damage that is induced by oxidative stress. However, there is a personal variation in ACE and ACE2 balance that clarifies different responses of COVID-19 patients and the various severity of cases that ranges from severe lethal condition to mild asymptomatic condition. Therefore, the prevention and treatment of COVID-19 infection should depend on the suppression of toxic oxidative stress as the best solution to get rid of this outbreak because of the ability of reactive oxygen species to induce pro-inflammatory cytokines creating the inflammation process that leads to acute respiratory distress syndrome and fulminant myocarditis [8].

\section{ENDOGENOUS ANTIOXIDANTS AND COVID-19 INFECTION}

At first, it should mention glutathione (GSH) as the main and essential cellular antioxidant in different body tissues that has an important cytoprotective role in the detoxification process and immune response via reducing hydrogen peroxide and lipid hydroperoxides protecting the body against reactive oxygen species. Glutathione consists of amino acids, glutamate, cysteine, and glycine wherein it is de novo synthesis or import from the lumen of the intestine via specific plasma membrane transporters. Depletion of glutathione may be caused by oxidation or a decrease in its formation as the hypoxic case that is an inhibitory agent for glutathione synthesis. On other hand, vitamin D can increase the level of glutathione via induction of gene expression of glutamate-cysteine ligase (GCL) and glutathione reductase whereas the GCL gene is suppressed by reactive oxygen species and nitric oxide [9].

Therefore, glutathione has the ability for preventing cellular damage that is caused by toxic oxidative stress that plays an important role in the release and activation of proinflammatory cytokine in COVID-19 infection. So, the consumption of glutathione in these cases means fatal exacerbated inflammatory disorders [10]. Furthermore, there is NF-kB factor that is considered one of the redox-sensitive transcription factors that have an important role in inflammation and pathological pulmonary changes in COVID-19 infection. In addition, 
activation of NF-kB is suppressed by glutathione leading to a limitation in inflammatory and pathological changes of the lung via controlling the cytokine storm wherein virus activates interleukin-6 expression via NF$\mathrm{kB}$ binding at the genetic region. Thus, any drug that can inhibit NF-kB activation will limit the harmful pathological effects of COVID-19 infection at the same time [11].

In the same context, there are many studies that confirmed the role of glutathione as an antioxidant in the treatment of COVID-19. Moreover, it was noted that most of the severe cases who have risk factors suffer also from a decrease in the level of glutathione such as diabetes, old age, hypertension, and obesity. Therefore, we can conclude that antioxidants can play a critical role in ameliorating the toxic effect of oxidative stress that is induced by COVID-19 infection [12].

Studies showed also that systemic oxidative stress is associated with a decrease in the total free thiol levels such as cysteine, homocysteine, and glutathione. Noteworthy, systemic free thiols levels can be affected via therapeutic intervention and nutrition. Thus, anti-oxidant therapy may be useful in any inflammation case related to impaired redox homeostasis such as COVID-19 infection. In a related context, Nacetylcysteine is considered one of the substrates in glutathione synthesis with antioxidant properties, so it is considered a promising therapeutic option in COVID-19 cases to restore toxidant -antioxidant balance modulating pulmonary inflammation [13]. Furthermore, N-acetylcysteine has a mucolytic effect besides it is also a glutathione precursor that inhibits viral replication. Therefore, oral administration of $\mathrm{N}$-acetylcysteine (NAC) may be used as a preventive measure for health professionals because of the repeated exposure to COVID-19 carriers. In addition, some clinical trials suggested that administration of 150 $\mathrm{mg} / \mathrm{kg}$ NAC as a bolus followed by another 50 $\mathrm{mg} / \mathrm{kg} /$ daily for 4 days in patients with respiratory problems may improve oxygenation and reduce mortality [14].

Moreover, oral administration of glutathione, or proteins containing amino acids that are constituents of glutathione can increase the concentration of glutathione in the body. In the related context, recent studies concluded that the repeated use of glutathione (oral and intravenous administration) can relieve the severe pulmonary manifestations of COVID-19 as an antioxidant therapeutic option in COVID-19 cases [15].

In another context, it is known that free iron in the blood and tissues can be easily oxidized to be a trigger to lipid peroxidation, so the binding of ferritin with iron ions can decrease oxidative damage in the tissues. Therefore, it was noted that there is a significantly high level of serum ferritin in COVID-19 cases as a compensatory mechanism to reduce the free iron level that is produced from the breakdown of hemoglobin induced by the virus. Worthwhile, iron metabolism is under control physiologically to maintain the redox balance and iron homeostasis in the body protecting the cell from toxicity. This control is found in pulmonary alveoli wherein macrophages can eliminate reactive free radicals associated with sufficient antioxidant molecules to neutralize them. Thus, the breakdown of hemoglobin and the resulting hypoxia inducing pulmonary injury in the cases of COVID-19 may be treated by the pyrimidine nucleoside uridine as a natural antioxidant and an antihypoxic agent wherein the use of uridine preserves the alveolar structure and the air blood barrier in the lung as an effective free radical scavenger [16].

NATURAL ANTIOXIDANTS AS AN Alternative Therapy FOR COVID-19 INFECTION

Based on the above-mentioned, we can use natural antioxidants as an alternative or an adjuvant therapeutic tool in treating COVID-19 cases such as flavonoids that are widely found in vegetables and fruits. Moreover, flavonoids include also quercetin, catechin, apigenin, luteolin, and rhamnoside which can inhibit the accumulation of reactive oxygen species and cell apoptosis that result from COVID-19 infection. In addition, several studies concluded that natural antioxidants have also an antiviral effect by preventing the replication of COVID-19. Therefore, the use of some plants or herbal medicine as an alternative or adjuvant treatment in COVID-19 cases may be useful because it contains these natural antioxidants such as green tea (high levels of catechins), Ginkgo biloba L., (high levels of amentoflavone), citrus fruits (high levels of Rhoifolin), carrot, cabbage, and apple (high levels of Luteolin), and grapes, peanuts, and blueberries (high levels of Resveratrol). According to the published literature, these above-mentioned natural antioxidants can increase the levels of 
antioxidant enzymes (superoxide dismutase and catalase) and non-enzymatic antioxidants (vitamins $\mathrm{C}, \mathrm{E}$, and glutathione) reducing oxidative stress and lipid peroxidation [17].

In the same context, polyphenols have also an anti-inflammatory effect besides their antioxidant and antiviral properties. Noteworthy, catechins are one of the polyphenolic flavonoids wherein it is plant-derived phenolic compounds that present in green tea leaves. Moreover, polyphenols are considered as prebiotics wherein it can sustain gut-lung axis integrity leading to protection against viral infections via improving immune response to viral infections such as COVID-19. In addition, the anti-inflammatory effect of polyphenols can combat the effect of cytokines storm in COVID-19 cases via impeding the maturation and migration of cytokines promoting its antiviral effect besides its ability to suppress the formation of angiotensin II-associated C-reactive protein that has an essential role in the inflammation progression [18].

Furthermore, polyphenols can reduce body weight altering the body composition leading to a disturbance in systemic and adipose tissue inflammation that is responsible for cytokine secretion in obese COVID-19 patients wherein obesity aggravates the viral outcomes as one of the risk factors in cases of COVID-19 infection. Polyphenols can also improve the other risk factors that cause a poor prognosis in COVID19 cases such as hypertension, diabetes, and dyslipidemia. However, although the ability of polyphenols to act synergistically with other drugs against COVID-19 infection, it has a limited bioavailability because it loses a considerable proportion during digestion whereas its absorbed part via the small intestine is small relatively [19].

In the related context, curcumin is also considered one of the polyphenolic compounds that can bind with the target receptor of COVID-19. Thus, curcumin is suggested as one of the preventive or therapeutic options in cases of COVID-19 infection. Furthermore, the combination of curcumin with vitamin $\mathrm{C}$ and glycyrrhizic acid can produce interferons modulating the inflammatory response in cases of COVID-19 infection according to Chen and his colleagues [20]. Hydroxytyrosol is also another phenolic compound found in extra virgin olive oil that has antioxidant, antiinflammatory, and antiviral effects attenuating the pulmonary fibrosis process, thus it may be useful in therapeutic protocols of COVID-19 [21].
On other hand, Vitamin C (ascorbic acid) is considered a non-enzymatic antioxidant that is a free radical scavenger in the human body preventing cell damage and protect the body from different diseases that are induced by oxidative stress such as atherosclerosis, arthritis, and cancer. Moreover, it was noted that the plasma level of vitamin $\mathrm{C}$ has an inverse proportion with the incidence rate of multiorgan failure in critical patients while it has a direct proportional with the survival rates. In addition, clinical trials revealed that vitamin $\mathrm{C}$ can decrease the incidence of lower respiratory tract infections such as pneumonia [22]. Furthermore, other studies indicated that vitamin $\mathrm{C}$ administration may reduce the duration of mechanical ventilation and then the length of stay in the intensive care unit, besides it can also minimize the need of the patient for vasopressor agents. Therefore, parenteral administration of high doses of vitamin $\mathrm{C}$ may be safe and effective as an alternative therapy for critical patients such as COVID-19 cases. Moreover, some clinical trials showed that the combination of vitamin $\mathrm{C}$ and $\mathrm{E}$ may be a promising therapeutic option that can decrease oxidative stress, subsequent pulmonary inflammation, and the organic damage which are caused by COVID-19 [23].

Other nutraceuticals may also play an important role in combating COVID 19 pandemic. Some clinical trials and researches suggested that high doses of vitamin D3 as one of the dietary supplements can prevent and treat COVID 19 infection besides other minerals such as zinc and selenium because of their antiviral and immunomodulatory properties through its redox homeostasis and antioxidant properties. Moreover, probiotic therapy as a nutritional antioxidant supplementation is considered beneficial for COVID 19 patients such as Lactobacillus acidophilus W22, Bifidobacterium lactis W51, and Bifidobacterium bifidum W23 according to the recommendation of the National Administration of Traditional Chinese Medicine and the Chinese National Health Commission. In addition, omega 3 fatty acids may also be useful and effective as an antiinflammatory agent reducing bronchoconstriction. Furthermore, other studies reported that melatonin as an antioxidant and anti-inflammatory agent can modulate the immune system leading to a better prognosis in COVID 19 cases [24]. 
Noteworthy, there are new suggested pharmacological therapeutic strategies in recent times but most of them are based on the same mechanism that depends on cytoprotection and anti-inflammatory effects via restoring redox homeostasis promoting the inflammation resolution. One of these strategies is pharmacological activation of nuclear factor erythroid $2 \mathrm{p} 45$-related factor2 (NRF2) that protects from COVID 19 infection such as sulforaphane and bardoxolone methyl which are considered electrophilic NRF2 activators. Moreover, NRF2 activation may persist for several days because it is mediated by enzymes that have long half-lives unlike other direct antioxidants such as vitamin $\mathrm{C}$ that may persist minutes to hours wherein it is consumed in the ROS scavenging process [25].

\section{How to Design AN EFFECTIVE Therapeutic Protocol For COVID 19 PATIENTS?}

At first, it should know that the efficacy of any therapeutic protocol for COVID 19 cases depends on the comorbidity condition of the patient at the time of infection, age, genetic base, and gender besides the possible side effects of the used drugs and their impact on the patient condition. Furthermore, delayed diagnosis and therapeutic intervention besides the time of the inflammatory evolution that triggered via COVID 19 infection may play role in the determination of the patient outcome. In addition, there are other factors related to the therapeutic agent such as the dose, the route of administration, the plasma concentration, the beginning and duration of treatment besides the heterogeneity response of the target patients. Moreover, the side effects of medications and their interaction if it is used as combinatorial therapy should be taken into account during the design of any therapeutic protocol besides other risk factors such as diabetes, hypertension, and obesity because of the higher mortality rate in these cases. So, the design of any therapeutic protocol for COVID 19 patients should take into consideration the above-mentioned factors [26].

In the same context, any therapeutic strategy should depend on two bases; the first is aimed to reduce the viral load while the second is aimed to prevent sepsis and multiple organ failure that are caused by the uncontrolled inflammatory process. Thus, this may be achieved via using antioxidant agents as antiviral besides its ability to reduce this inflammatory storm at the same time preventing the bad outcome for the patients. [27].

In addition, other benefits of antioxidant therapy have been discovered wherein it can improve oxygenation and immune response leading to a time reduction for the mechanical ventilation lowering the length of stay in intensive care and hospital associated with a decrease in the rate of multiple organs failure and mortality in COVID 19 patients [28].

In this context and since there is no proven treatment for COVID 19 yet, so the use of alternative and adjuvant antioxidant treatment becomes an urgent necessity because it is considered useful for the human body without causing any harmful effect. However, it should conduct further studies and clinical trials on the human to verify its preventive and therapeutic efficacy against COVID 19 infection [29].

\section{CONCLUSION}

Toxic oxidative stress is responsible for the pathogenesis of COVID -19 infection wherein the main life-threatening pathological feature of COVID-19 is lung inflammation that results from the lethal cytokine storm. Therefore, the imbalance between oxidant agents and the antioxidant system can lead to an increase in free radicals production concomitant with antioxidants depletion inducing toxic oxidative stress in COVID-19 infection. However, the toxic effect of these free radicals may be neutralized by endogenous and natural antioxidants such as glutathione, vitamins $\mathrm{C}$ and $\mathrm{E}$, enzymes such as glutathione peroxidase, superoxide dismutase, and catalase, herbal medicine and other nutraceuticals, besides some trace elements such as zinc and selenium preventing the pathological features of Covid-19. Regarding there is no specific therapy for COVID-19 cases until now, there are many attempts to design therapeutic protocols based on the effect of antioxidants as a complementary and alternative treatment. So, it should evaluate the efficacy of these therapeutic options via conducting further clinical trials.

\section{REFERENCES}

[1] Wenxiao T, Houlin T, Fangfang C, Yinong W, Tingling X, et al (2020) Epidemic update and risk assessment of 2019 Novel Coronavirus. Chinese Center for Disease Control and Prevention, 2(6): 83-86.

[2] Camini FC, Caetano CC, Almeida LT, De Brito Magalhães C (2017) Implications of Oxidative Stress on Viral Pathogenesis. Arch Virol; 162 (4):907-917. 
[3] Zhang Z, Rong L, Li YP (2019) Flaviviridae Viruses and Oxidative Stress: Implications for Viral Pathogenesis. Oxid Med Cell Longev; 2019:1409582. DOI: http://doi.org/10.1155/20 19/1409582

[4] Fraternale A, Paoletti MF, Casabianca A, Nencioni L, Garaci E, Palamara AT, et al (2009) GSH and analogs in antiviral therapy. Molecular Aspects of Medicine, 30(1-2):99-110.

[5] Akaike T (2001) Role of free radicals in viral pathogenesis and mutation. Rev Med Virol; 11 (2):87-101.

[6] Zeliger HI, Kahaner H (2020) Can the Oxidative Stress Index Predict the Severity of COVID-19? European Journal of Medical and Health Sciences, 2(2): 1-5. DOI: http://dx.doi. org/10.24018/ejmed.2020.2.2.233

[7] Kuba K, ImaiY, Rao S, Gao H, Guo F, Guan B, Huan Y, Yang P, Zhang Y, Deng $\mathrm{W}$ et al (2005) A crucial role of angiotensin converting enzyme 2 (ACE2) in SARS coronavirusinduced lung injury. Nat Med; 11: 875- 879.

[8] Vaduganathan M, Vardeny $\mathrm{O}$, Michel $\mathrm{T}$, McMurray JJV, Pfeffer MA, Solomon SD (2020) Renin-Angiotensin-Aldosterone System Inhibitors in Patients with Covid-19. N Engl J Med; 382: 1653- 1659.

[9] Reid M, Jahoor F (2001) Glutathione in disease. Curr Opin Clin Nutr Metab Care; 4: 65-71.

[10] Basi Z, Turkoglu V (2019) In vitro effect of oxidized and reduced glutathione peptides on angiotensin converting enzyme purified from human plasma. J Chromatogr B; 1104: 190-195.

[11] Xu Z, Shi L, Wang Y, Zhang J, Huang L, Zhang C et al (2020) Pathological findings of COVID-19 associated with acute respiratory distress syndrome. The Lancet Respiratory Medicine, 8(4):420-422.

[12] Andrich DE, Melbouci L, Ou Y, Auclair N, Mercier J, Grenier JC, Lira FS, Barreiro LB, Danialou G, Comtois AS, et al (2019) A ShortTerm High-Fat Diet Alters Glutathione Levels and IL-6 Gene Expression in Oxidative Skeletal Muscles of Young Rats. Front Physiol; 10: 372.

[13] Polonikov A (2020) Endogenous Deficiency of Glutathione as the Most Likely Cause of Serious Manifestations and Death in COVID19 Patients. ACS Infect Dis; 6: 15581562.

[14] Sanguinetti CM (2016) N-acetylcysteine in COPD: Why, how, and when? Multidiscip Respir Med; 11.

[15] Janssen-Heininger Y, Reynaert NL, van der Vliet A., Anathy V (2020) Endoplasmic reticulum stress and glutathione therapeutics in chronic lung diseases. Redox Biol; 33:101516.

[16] Mironova GD, Belosludtseva NV, Ananyan MA (2020) Prospects for the use of regulators of oxidative stress in the comprehensive treatment of the novel Coronavirus Disease
2019 (COVID-19) and its complications. European Review for Medical and Pharmacological Sciences; 24: 8585-8591

[17] Diniz LR L, Filho CSB, Fielding BC, de Sousa DP (2020) Natural Antioxidants: A Review of Studies on Human and Animal Coronavirus. Oxidative Medicine and Cellular Longevity, Article ID 3173281. DOI: https://doi.org/ $10.1155 / 2020 / 3173281$

[18] Levy E, Delvin E, Marcil V, Spahis S (2020) Can phytotherapy with polyphenols serve as a powerful approach for the prevention and therapy tool of novel coronavirus disease 2019 (COVID-19)? Am J Physiol Endocrinol Metab; 319: 689 -708.

[19] Li D, Zhang T, Lu J, Peng C, Lin L (2020) Natural constituents from food sources as therapeutic agents for obesity and metabolic diseases targeting adipose tissue inflammation. Crit Rev Food Sci Nutr; 28: 1-19. DOI:10.1080/10408398.2020.1768044.

[20] Chen L, Hu C, Hood M, Zhang X, Zhang L, Kan J, Du J (2020) A novel combination of vitamin $\mathrm{C}$, curcumin and glycyrrhizic acid potentially regulates immune and inflammatory response associated with coronavirus infections: A perspective from system biology analysis. Nutrients, 12(4): 1193. DOI: https://doi.org/10.3390/nu120 41193

[21] Lammi C, Bellumori M, Cecchi L, Bartolomei M, Bollati C, Clodoveo ML, Nadia M (2020) Extra virgin olive oil phenol extracts exert hypocholesterolemic effects through the modulation of the LDLR pathway. In vitro and cellular mechanism of action elucidation. Nutrients, 12(6): 1723

[22] Al- Taie A, Victoria AO (2020) Supplementary medicines and antioxidants in viral infections: A review of proposed effects for COVID- 19. Biomed Biotechnol Res J; 4:19-24.

[23] Alan Rossetti C, Real JP, Daniel Palma S (2020) High Dose Of Ascorbic Acid Used In Sars Covid-19 Treatment: Scientific And Clinical Support For Its Therapeutic Implementation. Ars Pharm; 61(2): 145-148.

[24] Tahir AH, Javed MM, Hussain Z (2020) Nutraceuticals and herbal extracts: $A$ ray of hope for COVID 19 and related infections (Review). INTERNATIONAL JOURNAL OF FUNCTIONAL NUTRITION, 1: 6.

[25] Cuadrado A, Pajares M, Benito C, JiménezVillegas J, Escoll M, Fernández-Ginés R, et al (2020) Can Activation of NRF2 Be a Strategy against COVID-19? Trends in Pharmacological Sciences, 41: (9):598-610. DOI: https://doi.org/10.1016/j.tips.2020.07.003

[26] Soto ME, Guarner-Lans V, Soria-Castro E, Pech LM, Pérez-Torres I (2020) Is Antioxidant Therapy a Useful Complementary Measure for 
Efficacy of Antioxidants in Amelioration the Toxic Effect of Oxidative Stress Induced By COVID- 19 Infection

Covid-19 Treatment? An Algorithm for Its Application. Medicina; 56:386. Doi: 10.3390/ medicina56080386

[27] Crimi E, Liguori A, Condorelli M, Cio M, Astuto M, Bontempo P, Pignalosa O, Vietri MT, Molinari AM, Sica V et al (2004) The beneficial e_ects of antioxidant supplementation in enteral feeding in critically ill patients: A prospective, randomized, double-blind, placebo -controlled trial. Anesth Analg; 99: 857- 863.
[28] Horowitz RI, Freeman PR, Bruzzese J (2020) Efficacy of glutathione therapy in relieving dyspnea associated with COVID-19 pneumonia: A report of 2 cases. Respir Med Case Rep; 30: 101063. DOI: 10.1016/j. rmcr. 2020.101063.

[29] Howe KP, Clochesy J.M, Goldstein LS, Owen $\mathrm{H}$ (2015) Mechanical ventilation antioxidant trial. Am. J Crit Care; 24:440-445.

Citation: Said Said Elshama, "Efficacy of Antioxidants in Amelioration the Toxic Effect of Oxidative Stress Induced By COVID- 19 Infection”, International Journal of Research Studies in Medical and Health Sciences. 2021; 6(2): 21-27. DOI: https://doi.org/10.22259/ijrsmhs.0602005

Copyright: (C) 2021 Said Said Elshama, This is an open-access article distributed under the terms of the Creative Commons Attribution License, which permits unrestricted use, distribution, and reproduction in any medium, provided the original author and source are credited. 